\title{
LA-UR- $02-7510$
}

Approved for public release: distribution is unlimited.

\section{Submitted to:}

\section{Space Technology \& Applications International Forum,} STAIF-2003

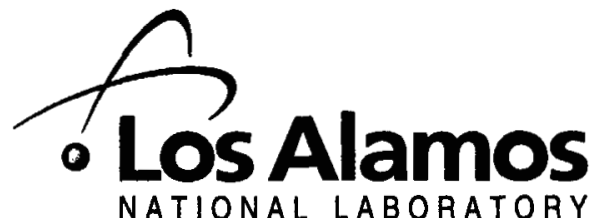

Los Alamos National Laboratory, an affirmative action/equal opportunity employer, is operated by the $U$. sity of California for the U.S. Department of Energy under contract W-7405-ENG-36. By acceptance of this article, the publisher recty. izes that the U.S. Government retains a nonexclusive, royalty-free license to publish or reproduce the published form of this contribution, or to allow others to do so, for U.S. Government purposes. Los Alamos National Laboratory requests that the publisher identify this article as work performed under the auspices of the U.S. Department of Energy. Los Alamos National Laboratory strongly supports academic freedom and a researcher's right to publish; as an institution, however, the Laboratory does not endorse the viewpoint of a publication or guarantee its technical correctness. 


\title{
Transient Thermohydraulic Heat Pipe Modeling: Incorporating THROHPUT into the CÆSAR Environment
}

\author{
Michael L. Hall \\ Los Alamos National Laboratory, P.O. Box 1663, MS-D409, Los Alamos, NM 87545 \\ Phone: 505-665-4312,Email: Hall@LANL.gov
}

\begin{abstract}
The THROHPUT code, which models transient thermohydraulic heat pipe behavior, is being incorporated into the CASAR computational physics development environment. The CÆSAR environment provides many beneficial features for enhanced model development, including levelized design, unit testing, Design by Contract ${ }^{\text {TM }}$ (Meyer, 1997), and literate programming (Knuth, 1992), in a parallel, object-based manner. The original THROHPUT code was developed as a doctoral thesis research code; the current emphasis is on making a robust, verifiable, documented, component-based production package. Results from the original code are included.
\end{abstract}

\section{THE THROHPUT CODE}

Heat pipes are structures that transport heat by the evaporation and condensation of a working fluid, giving them a high effective thermal conductivity. Many space-based uses for heat pipes have been suggested, and high temperature heat pipes using liquid metals as working fluids are especially attractive for these purposes. These heat pipes are modeled by the THROHPUT code (THROHPUT is an acronym for Thermal Hydraulic Response Of Heat Pipes Under Transients and is pronounced the same as the word "throughput").

\section{Summary Description of THROHPUT}

The THROHPUT code provides a detailed model of the transient, thermohydraulic behavior of liquid metal heat pipes. Currently, it models homogeneous or composite wicks in a cylindrical heat pipe structure. The boundary conditions assumed are: a time-dependent heat input in the evaporator section, zero heat transfer at the outer wall in the adiabatic section, and radiative cooling to a specified ambient temperature in the condenser section. The user specifies the dimensions of the heat pipe and of the wick structure.

The THROHPUT code models the transport and exchange of all three phases of the working fluid (solid, liquid, and gas) and of a noncondensible gas. It considers axial and radial conduction, axial and radial convection, axial and radial phase change heat transfer (both evaporation/condensation and melting/freezing) and radiative heat transfer from the condenser section. It has a geometric model for the capillary pressure difference generated by the wick. To model all of these parameters, THROHPUT solves a system which consists of fifteen partial differential equations at each axial node. The associated algebraic system consequently has a block tridiagonal matrix structure with $15 \times 15$ blocks and a set of blocks for each node. The temporal scheme used is a fully-implicit scheme which iterates on the Jacobian matrix until convergence. A sophisticated time step control scheme is utilized.

\section{Axial Model Equation Set}

Currently, THROHPUT uses the area-averaged Navier-Stokes equations to model the behavior of the working fluid (solid, liquid and gas) and the noncondensible gas. Submodels are used to calculate the effects of interphasic transfer processes (Radial Model), diffusive terms, friction loss terms, state equations, the axial capillary force term, and the capillary pressure relationship (Surface Model). All of these submodels are formulated in such a way that they can be inserted into the Axial Model equation set and solved simultaneously, in a fully implicit manner. 
The Axial Model equation set models the overall thermohydraulic behavior of the heat pipe system using fifteen separate equations at each node. These equations are:

\section{Continuity (Conservation of Mass) Equations}

Mixture of Gases (Working Fluid and Noncondensible) Continuity:

$$
\frac{\partial}{\partial t}\left(\alpha_{m} \rho_{m}\right)+\frac{\partial}{\partial z}\left(\alpha_{m} \rho_{m} V_{m}\right)=\sum_{x=l, s} \Gamma_{x g}
$$

Noncondensible Continuity:

$$
\frac{\partial}{\partial t}\left(\alpha_{m} \rho_{m} X_{n}\right)+\frac{\partial}{\partial z}\left(\alpha_{m} \rho_{m} X_{n} V_{m}\right)=\frac{\partial}{\partial z}\left(\alpha_{m} D_{n}^{X} \frac{\partial X_{n}}{\partial z}\right)+\frac{\partial}{\partial z}\left(\alpha_{m} D_{n}^{\rho} \frac{\partial \rho_{m}}{\partial z}\right)
$$

Liquid Continuity:

$$
\epsilon_{v} \frac{\partial}{\partial t}\left(\alpha_{l} \rho_{l}\right)+\epsilon_{v} \frac{\partial}{\partial z}\left(\alpha_{l} \rho_{l} V_{l}\right)=\sum_{x=g, s} \Gamma_{x l}
$$

Solid Continuity:

$$
\epsilon_{v} \frac{\partial}{\partial t}\left(\alpha_{s} \rho_{s}\right)=\sum_{x=g, l} \Gamma_{x s}
$$

\section{Conservation of Internal Energy Equations}

Mixture of Gases (Working Fluid and Noncondensible) Internal Energy:

$$
\begin{aligned}
& \frac{\partial}{\partial t}\left(\alpha_{m} \rho_{m} U_{m}\right)+\frac{\partial}{\partial z}\left(\alpha_{m} \rho_{m} U_{m} V_{m}\right)=-P_{m}\left(\frac{\partial}{\partial z}\left(\alpha_{m} V_{m}\right)+\frac{\partial \alpha_{m}}{\partial t}\right) \\
& +\frac{\partial}{\partial z}\left(\alpha_{m} D_{n}^{X}\left(h_{n}-h_{g}\right) \frac{\partial X_{n}}{\partial z}\right)+\frac{\partial}{\partial z}\left(\alpha_{m} D_{n}^{\rho}\left(h_{n}-h_{g}\right) \frac{\partial \rho_{m}}{\partial z}\right) \\
& +\frac{\partial}{\partial z}\left(\alpha_{m} k_{m} \frac{\partial T_{m}}{\partial z}\right)+\sum_{x=l, s}\left(Q_{x m}+Q_{x g}^{\Gamma}\right)
\end{aligned}
$$

Liquid Internal Energy:

$$
\begin{aligned}
& \epsilon_{v} \frac{\partial}{\partial t}\left(\alpha_{l} \rho_{l} U_{l}\right)+\epsilon_{v} \frac{\partial}{\partial z}\left(\alpha_{l} \rho_{l} U_{l} V_{l}\right)=-\epsilon_{v} P_{l}\left(\frac{\partial \alpha_{l} V_{l}}{\partial z}+\frac{\partial \alpha_{l}}{\partial t}\right) \\
& +\epsilon_{v} \frac{\partial}{\partial z}\left(\alpha_{l} k_{l} \frac{\partial T_{l}}{\partial z}\right)+\sum_{x=m, s, w} Q_{x l}+\sum_{x=g, s} Q_{x l}^{\Gamma}
\end{aligned}
$$

Solid Internal Energy:

$$
\epsilon_{v} \frac{\partial}{\partial t}\left(\alpha_{s} \rho_{s} U_{s}\right)=\epsilon_{v} \frac{\partial}{\partial z}\left(\alpha_{s} k_{s} \frac{\partial T_{s}}{\partial z}\right)+\sum_{x=m, l, w} Q_{x s}+\sum_{x=g, l} Q_{x s}^{\Gamma}
$$

Wall Internal Energy:

$$
\rho_{w} c_{p_{w}} \frac{\partial T_{w}}{\partial t}=\frac{\partial}{\partial z}\left(\alpha_{w} k_{w} \frac{\partial T_{w}}{\partial z}\right)+Q_{i n}+\sum_{x=l, s} Q_{x w}
$$

\section{Conservation of Momentum Equations}

Mixture of Gases (Working Fluid and Noncondensible) Momentum:

$$
\frac{\partial}{\partial t}\left(\alpha_{m} \rho_{m} V_{m}\right)+\frac{\partial}{\partial z}\left(\alpha_{m} \rho_{m} V_{m}^{2}\right)=-\alpha_{m} \frac{\partial P_{m}}{\partial z}-\mathcal{F}_{m} V_{m}+\alpha_{m} \rho_{m} g_{z}
$$

Liquid Momentum:

$$
\epsilon_{v} \frac{\partial}{\partial t}\left(\alpha_{l} \rho_{l} V_{l}\right)+\epsilon_{v} \frac{\partial}{\partial z}\left(\alpha_{l} \rho_{l} V_{l}^{2}\right)=-\epsilon_{v} \alpha_{l} \frac{\partial P_{l}}{\partial z}-\mathcal{F}_{l} V_{l}-\Delta P_{c a p}^{\nu} \frac{\partial \alpha_{l}}{\partial z}+\epsilon_{v} \alpha_{l} \rho_{l} g_{z}
$$




\section{Thermodynamic State Equations}

Mixture of Gases (Working Fluid and Noncondensible) State:

$$
P_{m}=\rho_{m} T_{m}\left(X_{n} R_{n}+\left(1-X_{n}\right) R_{g}\right)
$$

Liquid State:

Solid State:

$$
\rho_{l}=\rho_{l}\left(P_{l}, T_{l}\right)
$$

$$
\rho_{s}=\rho_{s}\left(T_{s}\right)
$$

\section{Closure Equations}

Volume Fraction Sum:

$$
\sum_{x=m, l, s} \alpha_{x}=1
$$

Capillary Pressure Relation:

$$
P_{m}-P_{l}=\mathcal{L}\left(\Delta P_{\text {cap }}\left(\alpha_{m}\right)\right)
$$

The system variables in these equations are: $\rho_{m}, \rho_{l}, \rho_{s}, X_{n}, \alpha_{m}, \alpha_{l}, \alpha_{s}, P_{m}, P_{l}, V_{m}, V_{l}, T_{m}, T_{l}, T_{s}$ and $T_{w}$. The interphase transfer terms $\left(\Gamma_{x y}, Q_{x y}, Q_{x y}^{\Gamma}\right)$ are all functions of $\rho_{m}, X_{n}, T_{m}, T_{l}, T_{s}$, and $T_{w}$ and are defined in the Radial Model. A volume-based porosity has been included in the appropriate terms of the liquid and solid equations to account for the volume taken up by the wick structure. The internal energies of the phases are expressed in terms of the temperatures using other state equations in the final model. Boundary conditions for the Axial Model are simply the specification of zero velocity and zero heat flux on both ends of the heat pipe.

The axial model equation set is spatially discretized using a staggered mesh, with cell-boundary values for cell-centered properties being donored according to the appropriate velocity. The temporal discretization in THROHPUT is done in a fully implicit manner, resulting in a nonlinear equation which is solved via a Newton iteration. The Jacobian system matrix is block tridiagonal, which allows many terms to be differenced implicitly without significantly adding to the complexity of the solution scheme. Multiple Newton iterations are taken through the Jacobian system to solve the nonlinear system.

\section{Radial Model}

The Radial Model is used to determine the radial temperature distributions in the vapor core, wick and wall regions. In this model, a parabolic radial temperature distribution is assumed in each region, and the constants for the equations are related to the system variables in the Axial Model Equation Set given above. The Radial Model is then solved concurrently with the Axial Model.

Various boundary conditions and constraints on the average temperature are used in the model: Interphase mass and heat transfer between all of the phases is modeled. Melting and freezing are modeled with a discontinuous heat flux at the liquid-solid boundary. Evaporation and condensation are modeled using an evaporation rate derived from the kinetic theory of gases (modified to account for the presence of a noncondensible gas). Gas-solid mass transfer is modeled in a similar manner. Convective heat transfer at the liquid-vapor or solid-vapor interface is modeled. Through the solid and the heat pipe wall, heat transfer takes place by means of conduction. At the external wall boundary, a radiative heat flux condition is used. In the liquid, conduction and convection are both included in the model.

\section{Surface Model}

The heat pipe concept is based on the recirculation of the condensate liquid to the evaporator, which would be impossible without the capillary pressure head supplied by the wick. The capillary pressure relationship is therefore of utmost importance in heat pipe modeling. This relationship,

$$
P_{m}-P_{l}=\Delta P_{c a p}=\frac{2 \sigma}{r_{c}},
$$


allows for axial counter flow of the liquid and the gas through the variation of $r_{c}$, the radius of curvature of the surface of the liquid-vapor interface.

In steady state, $r_{c}$ varies from a value of infinity, which represents a flat surface, in the condenser to a small value in the evaporator section. The radius of curvature is strictly tied to the amount of liquid present. There will be a flat surface, $r_{c}=\infty$, if there is enough liquid present to cover or overflow the wick. As the liquid recedes into the wick, the radius of curvature becomes smaller and smaller until it reaches the limiting value of the pore radius in the wick. This condition usually exists in the evaporator.

In order to make Equation 16 as implicit as possible, the radius of curvature is related to a system variable, the gas mixture.volume fraction $\alpha_{m}$, using geometric arguments. The resulting equation is linearized to yield an implicit representation of the capillary pressure relationship that is included in the Axial Model Equation Set. For a full description of the wick surface model, see Hall (1991) or Hall (1988).

\section{Later Modifications to THROHPUT}

Early code runs demonstrated insufficient liquid return to the evaporator section, and the eventual dry-out of the evaporator section did not agree with experimental observations. One of the methods which was employed to counteract this problem was variation of the evaporation and condensation accommodation coefficients, but this alone was insufficient (Hall, 1988; Hall and Doster, 1990).

Further research into two areas yielded important information that made a significant impact on the modeling effort (Hall, Merrigan and Reid, 1994). First, research into experimental accommodation coefficient determination suggested that values closer to unity were appropriate for heat pipe conditions (Niknejad and Rose, 1981). Second, research into the possibility of liquid tension, or negative.liquid pressure, produced several references which validated its physical existence via experimentation. Liquid tension was originally mentioned in Berthelot (1850), and later multiple references are given in Hayward (1970) and Apfel (1972). Also, negative liquid pressure in heat pipes is treated specifically in Kemme (1968) and Anderson (1993), and experiments using Alkali Metal Thermal to Electric Converter (AMTEC) cells are presented in Anderson (1992) and Anderson et al. (1993). Subsequent to this research, changes were made to the capillary pressure relationship in THROHPUT to allow negative liquid pressure.

The original THROHPUT code was developed with only one working fluid (lithium), one wall material (molybdenum) and one noncondensible gas (air). Adding new materials involves locating and incorporating functional forms for thermal conductivity, specific heat, density, enthalpy, internal energy, viscosity, molecular weight and critical point variables. With later modifications, the THROHPUT code can now model several working fluids (lithium, sodium, potassium, rubidium, cesium, mercury and silver), two wall materials (molybdenum and rhenium) and one noncondensible gas (air).

\section{Results}

With the aforementioned modifications to THROHPUT the calculated response to heat pipe transients has been greatly improved. In particular, the liquid return to the evaporator section has been enhanced to the extent that during reasonable transients it is no longer a consideration.

THROHPUT has been used to model the transient operation of a SPAR-8 heat pipe which was fabricated and tested at Los Alamos National Laboratory (Merrigan, Keddy and Sena, 1986). This heat pipe had an evaporator section of $0.4 \mathrm{~m}$, an adiabatic section of $0.09 \mathrm{~m}$, and an overall length of $4.0 \mathrm{~m}$. It had an annular wick design and used lithium as a working fluid. The initial condition was a frozen solid state at $300 \mathrm{~K}$.

Unfortunately, there is a fundamental problem associated with modeling this experiment: the only available data from the experiment is the heat output, rather than the heat input. Heat pipe modeling codes in general, and THROHPUT in particular, need the heat input as a boundary condition. There is no way to use the heat output, which has been integrated over the entire condenser section, as a boundary condition for the code - for one thing the distribution in space is unknown. For the heat input boundary condition, it is assumed that the heat flux is evenly distributed over the evaporator section, which is a reasonable assumption. However, a constant heat flux assumption in the condenser section would be gravely wrong, since partial length operation, with distinctly nonconstant temperature profiles, is the standard start-up behavior. 


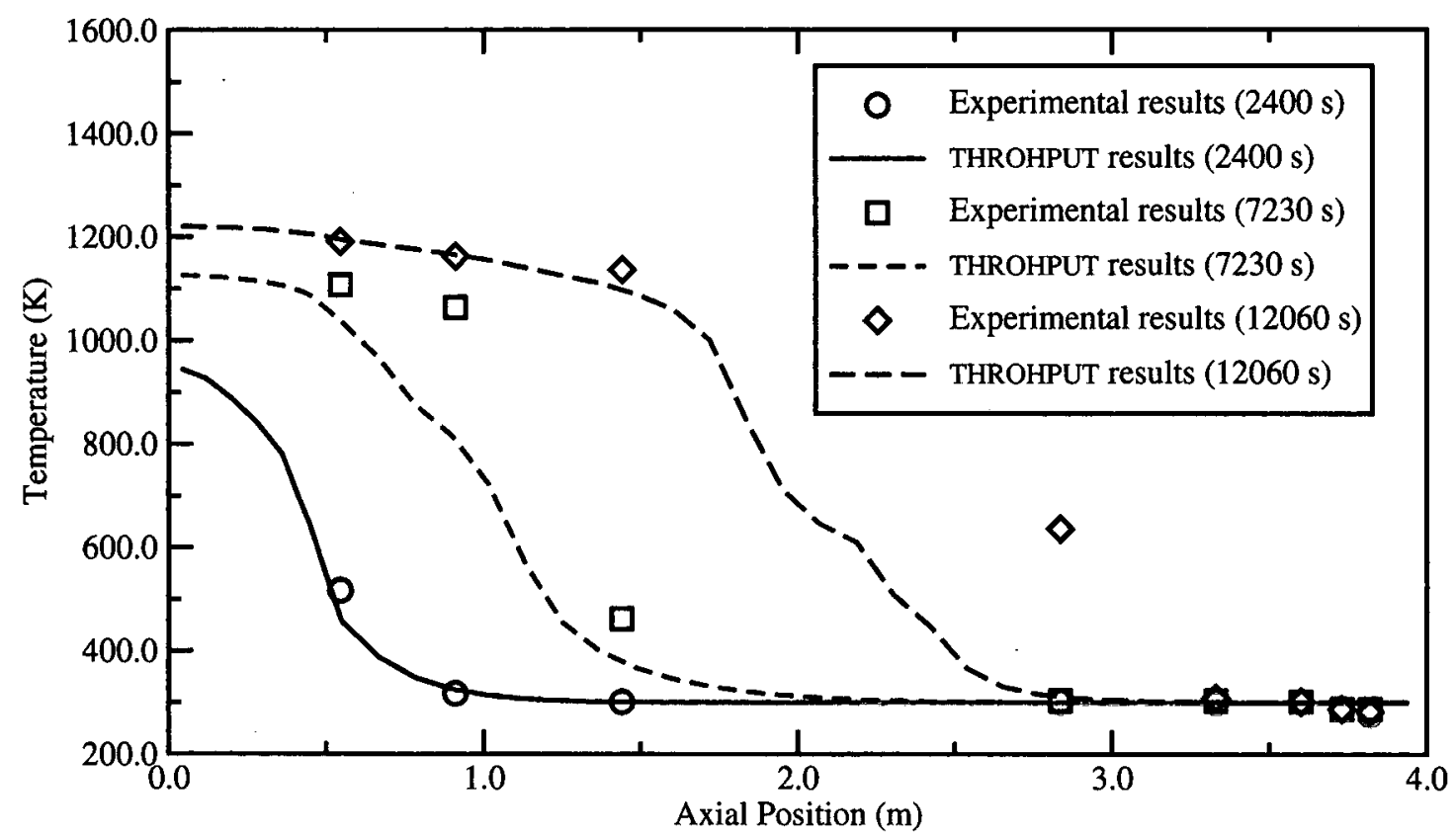

FIGURE 1. SPAR-8 Experimental Comparison (Merrigan et al., 1986) to THROHPUT Model Results at $2400 \mathrm{~s}$, $7230 \mathrm{~s}$, and $12060 \mathrm{~s}$. The THROHPUT Model used the experimental heat output as a heat input boundary condition.

The approach taken in this paper is to use the experimental heat output as the heat input boundary condition for modeling purposes. This is certainly wrong, but short of multiple runs with guessed inputs iterating to match the known output, it is the best option available. In the following, both raw and "cooked" results from this methodology are shown.

Figure 1 shows a comparison of the experimental measurements of the external wall temperature and the raw model results at three different times during the transient. At $2400 \mathrm{~s}$, there is very good agreement between the experimental and calculated values. At later times, the calculated values seem to lag behind the experimental values. The probable cause for this behavior is the problem mentioned above, namely that the values used for the heat input to the THROHPUT code were actually the experimentally measured heat output values. Since there is a time lag between heat input and output, a corresponding time lag is caused by using the output values instead of the real input values.

One way to deal with the time lag problem in a heuristic manner is as follows: for both the experimental and THROHPUT model situations, calculate a time-integrated total heat output,

$$
Q_{\text {int }}(t)=\int_{0}^{t} Q_{\text {out }}\left(t^{\prime}\right) d t^{\prime} .
$$

Then, for a given experimental time, determine the time in the THROHPUT model when the two $Q_{\text {int }}(t)$ values are equal. This technique assures that both experiment and calculation have output the same amount of heat from the condenser section of the heat pipe, but it is still fundamentally incorrect due to the different lengths of time seen by the model and the experiment. Using this heuristic technique, model-experiment agreement later in the transient is significantly improved (see Figure 2), but the good agreement early in the transient degrades.

Figure 3(a) shows the liquid and vapor pressures at the end of the modeled transient, $13,260 \mathrm{~s}$ ( $3.68 \mathrm{hrs})$. At this time in the model, the melt front is located roughly halfway down the length of the heat pipe, which is in partial length operation. On the condenser side of the melt front, all of the pressures are nearly zero, as the saturation pressure is almost zero at low temperatures. On the evaporator side of the melt front, a complete heat pipe cycle is in operation, with adequate liquid return to replenish the evaporative losses. Note that the liquid pressure gradient (and consequently liquid flow) would be non-existent without the ability to put the liquid in tension. The maximum tension in the liquid obtained here is $-150 \mathrm{~Pa}$. During transients that have been modeled to date, 


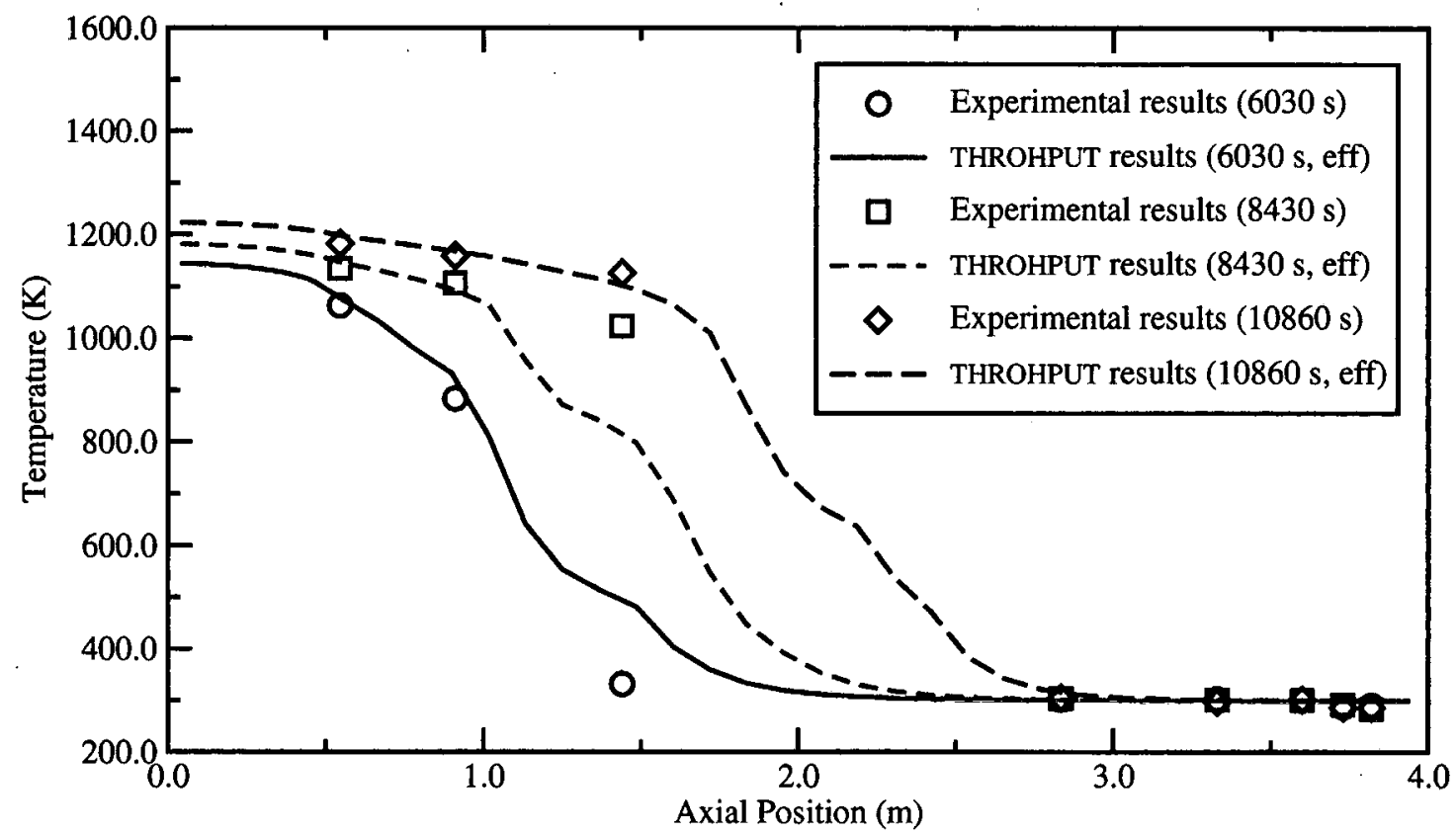

FIGURE 2. SPAR-8 Experimental Comparison (Merrigan et al., 1986) to Time-Adjusted THROHPUT Model Results at $6030 \mathrm{~s}, 8430 \mathrm{~s}$, and $10860 \mathrm{~s}$. The THROHPUT Model used the experimental heat output as a heat input boundary condition, and output times were adjusted (denoted as "eff") to match experimental time-integrated heat output.

the liquid pressure values have been calculated to be as low as $-10,000 \mathrm{~Pa}$, well within experimental observations and theoretical predictions.

The velocities of the liquid (multiplied by 10,000 ) and the vapor at $13,260 \mathrm{~s}$ are shown in Figure 3(b). The liquid velocity is negative, representing return flow toward the evaporator section. The vapor velocity curve is typical for heat pipes, increasing roughly linearly through the evaporator section, and then decreasing through the condenser section. The liquid velocity distribution is also consistent with expectations. Assuming that there is an approximate equilibrium in the mass flow rates of the vapor and liquid leads to a velocity ratio of 53,000 , which is indeed the case.

\section{THE CESAR ENVIRONMENT}

CASAR is a computational physics development environment; that is, it provides an environment where the physics of real systems can be modeled, by discretizing a set of partial differential equations on a mesh and solving the resultant algebraic system. It provides a consistent means of incorporating new methods of attacking computational physics problems. It is extensible - new equation sets, new discretizations, new meshes, new linear solvers, new communication libraries, etc., may be incorporated easily.

The emphasis in C\&SAR is on equation sets, discretizations, meshes, nonlinear solvers, and preconditioners, which are all incorporated into the basic CASAR structure. In contrast, linear solvers, communications libraries, mesh generators/partitioners, and visualization tools are generally included as external packages developed elsewhere.

CESAR is written in Fortran-90 (preprocessed by Gnu m4) in an object-based fashion, and probably comes as close to being object-oriented as is possible in Fortran-90. It has both parallel and serial versions, designed in from the start of the project. CESAR has a completely levelized design (Lakos, 1996); there are no dependency loops between classes or modules. It uses its own form of Design by Contract ${ }^{\mathrm{TM}}$ (Meyer, 1997) to verify the behavior of all procedures. CÆSAR uses extensive unit testing to certify all classes. It uses the ideas of literate programming (http://www.1iterateprogramming.com/) (Knuth, 1992) to generate documentation (in HTML, PostScript and PDF) from comments included in the code, via the Document Package (http://www.lanl.gov/Document). The 


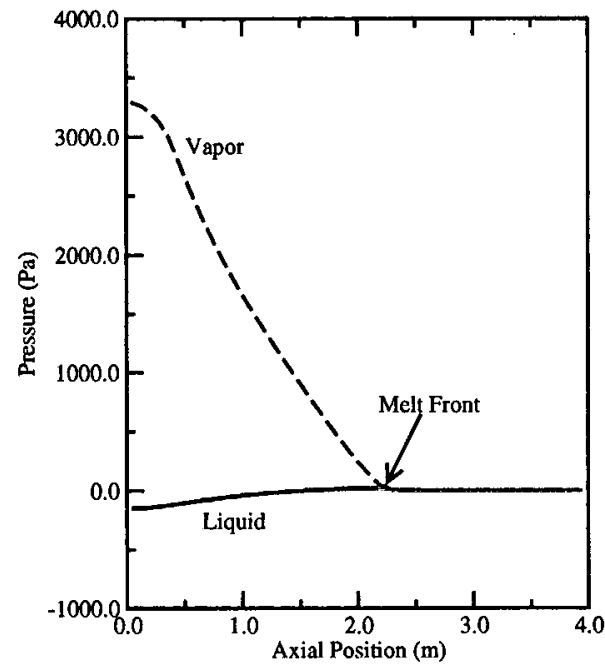

(a) Liquid and Vapor Pressure Distributions.

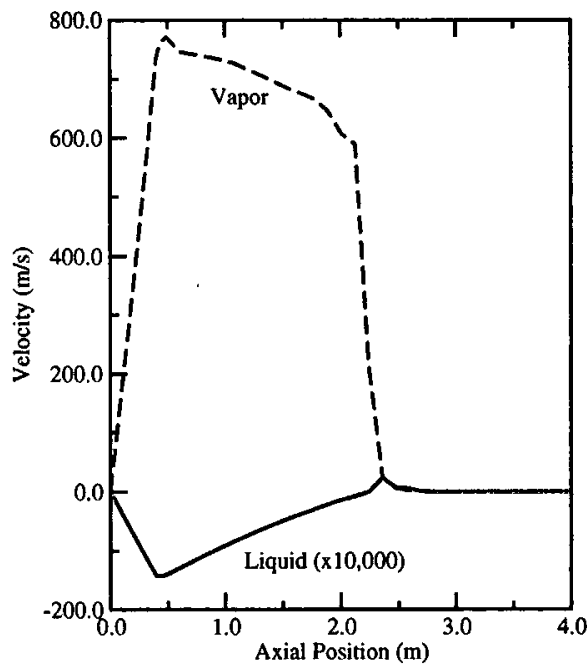

(b) Liquid and Vapor Velocity Distributions.

FIGURE 3. SPAR-8 THROHPUT Model Results at 13,260 s (3.68 hrs).

CASAR computational physics environment incorporates multiple mesh types, dimensionalities, and geometries. It supports multiple physics packages by allowing for different sets of partial differential equations.

\section{CÆSAR - THROHPUT Integration}

The next step in the evolution of the THROHPUT Code is integration into the CESAR Environment. Positioning THROHPUT as a package under the CESAR aegis will allow it to make use of all of the lower level data structures and methods in CASAR. Additionally, THROHPUT will be able to take advantage of the software construction features of CÆSAR: object-based design, unit testing, levelized design, Design by Contract $^{\mathrm{TM}}$, and documentation via literate programming.

After CASAR/THROHPUT integration is complete, research will be directed into modeling alternate wick geometries, such as axial groove and arterial wick designs. The THROHPUT code will continue to be used to predict heat pipe response to extreme transients, to analyze the merit of possible design alternatives, and to examine the sensitivity of heat pipe operation to physical phenomena.

\section{NOMENCLATURE}
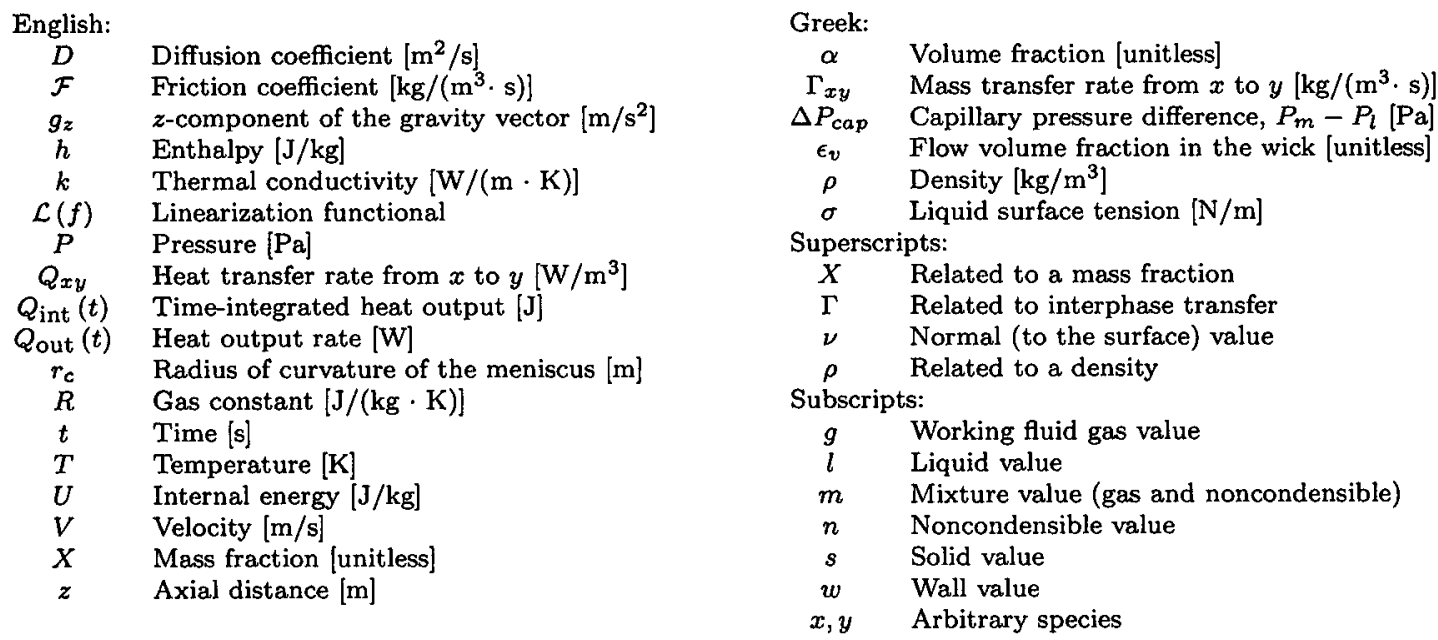


\section{REFERENCES}

Anderson, William G., "Sodium Wick Pumping Experiments for a Vapor-Fed AMTEC System", in Proceedings of the 27th Intersociety Energy Conversion Engineering Conference, Vol. 3, San Diego, CA, August 3-7 1992, pp. 111-116, paper no. 929146.

Anderson, William G., "Negative Pressures in Alkali Metal Heat Pipes", in Proceedings of the National Heat Transfer Conference, Atlanta, GA, August 8-11 1993.

Anderson, William G., Joseph J. Bland, Joseph F. Ivanenok and Robert K. Sievers, “Design of a Wick-Pumped, Vapor-Fed AMTEC System", in Proceedings of the 28th Intersociety Enengy Conversion Engineering Conference, Vol. 1, Atlanta, GA, August 8-13 1993, pp. 835-841, paper no. 93038 .

Apfel, Robert E., "The Tensile Strength of Liquids", Scientific American pp. 58-71, December 1972.

Berthelot, Marcellin, "Sur Quelques Phénomènes de Dilatation Forcée Des Liquides", Annales de Chimie et de Physique 30:232-237, 1850. An English translation is available online at http://THROHPUT. com.

Hall, Michael L., Numerical Modeling of the Transient Thermohydraulic Behavior of High Temperature Heat Pipes for Space Reactor Applications, PhD thesis, North Carolina State University, Department of Nuclear Engineering, Raleigh, NC.

Hall, Michael L., "Wick Surface Modeling in Heat Pipes", in Transactions of the 1991 American Nuclear Society Winter Meeting, San Francisco, CA, November 10-15 1991, pp. 735-737, LA-UR-91-1208.

Hall, Michael L. and J. Michael Doster, "A Sensitivity Study of the Effects of Evaporation/Condensation Accommodation Coefficients on Transient Heat Pipe Modeling", International Journal of Heat and Mass Transfer 33(3):465-481, March 1990.

Hall, Michael L., Michael A. Merrigan and Robert S. Reid, "Status Report on the THROHPUT Transient Heat Pipe Modeling Code", in Proceedings of the Eleventh Symposium on Space Nuclear Power and Propulsion, Albuquerque, NM, January 9-13 1994, pp. 965-970, LA-UR-93-3837; CONF-940101-17. Available online at http://THROHPUT. com.

Hayward, Alan T. J., "New Law For Liquids: Don't Snap, Stretch!", New Scientist pp. 196-199, January 291970.

Kemme, Joseph E., "Ultimate Heat Pipe Performance", in Proceedings of the Thermionic Conversion Specialist Conference, Framingham, MA, October 21-23 1968, pp. 266-271.

Knuth, Donald E., Literate Programming, (Stanford, California: Center for the Study of Language and Information), March 1992. CSLI Lecture Notes, No. 27, ISBN 0-937073-80-6. Online information is available at http://www-cs-facuity. stanford. edu/"knuth/lp.html.

Lakos, John, Large-Scale C++ Software Design, Addison Wesley, 1996. ISBN 0-201-63362-0.

Merrigan, Michael A., Edward S. Keddy and J. T. Sena, "Transient Performance Investigation of a Space Power System Heat Pipe", in Proceedings of the AIAA/ASME Fourth Joint Thermophysics and Heat Transfer Conference, AIAA-86-1273, Boston, MA, June 2-4 1986, LA-UR-86-1567.

Meyer, Bertrand, Object-Oriented Software Construction, second edn, ISE Inc., 1997. ISBN 0-13-629155-4. Online information is available at http://archive. $\theta$ iffel.com/doc/oosc/page.html.

Niknejad, J. and J. W. Rose, "Interphase Matter Transfer: An Experimental Study of Condensation of Mercury", Proceedings of the Royal Society of London 378:305-327, October 261981. 